\title{
Research on Explosive Separation of Carbon Fiber Composite Weave Plate
}

\author{
Zhijie He $\mathbb{D}^{1},{ }^{1}$ Meng Wang $\mathbb{D}^{1},{ }^{1}$ Kang Zhao, ${ }^{1}$ Hong Su, ${ }^{1}$ Zekan He, ${ }^{2}$ and Haijun Xuan ${ }^{2}$ \\ ${ }^{1}$ School of Chemical Engineering, Anhui University of Science and Technology, Huainan, 232000, China \\ ${ }^{2}$ School of Energy Engineering, Zhejiang University, Hangzhou 310000, China \\ Correspondence should be addressed to Meng Wang; 2019200639@aust.edu.cn
}

Received 8 December 2020; Revised 27 June 2021; Accepted 7 July 2021; Published 20 July 2021

Academic Editor: Amr A. Nassr

Copyright (c) 2021 Zhijie He et al. This is an open access article distributed under the Creative Commons Attribution License, which permits unrestricted use, distribution, and reproduction in any medium, provided the original work is properly cited.

By using the C-shaped metal lead shell and copper shell to wrap the detonating cord, the restraint of the explosion energy is improved, and the simple charge structure is used to achieve the purpose of effectively separating the carbon fiber composite woven plate in a limited and small space. The commercial software AUTODYN was used for numerical simulation, The monitoring points of stress, velocity, and specific internal energy were set up, combined with Tsai-Wu tensor strength criterion, the plate failure and the process of shell metal reflecting and absorbing energy when the plate was separated under the shell constraint were observed, respectively. Through further analysis of the experimental phenomena and calculated data, it is concluded that the main reason for the fracture failure of the plate is the opposing compression of the shock waves on both sides of the plate, and the layer cracks are caused by the tension of the stress wave in the later stage. The charge structure of the detonating cord wrapped by the metal shell can effectively use its inertia to restrain the explosion energy and improve the separation ability during the process of explosively separating the carbon fiber composite woven plate.

\section{Introduction}

Separation technology is a key link in aerospace engineering. At present, the separation technology mainly includes shaped charge cable [1], cylinder type explosive cable, and expansion pipe [2]. These separation devices are mainly composed of pyrotechnical components, which do not bear large loads themselves and have the advantages of simple structure, reliability, speediness, good synchronization, and so on. Therefore, they are widely used in the stage separation of many spacecraft at home and abroad. Hsiao [3] studied the characteristics of shaped charge jets using the finite element method. Xiong et al. [4] designed a three-dimensional (3D) finite element model of pyrotechnic cutters by using AUTODYN finite element simulation software and studied the influence of explosion dynamic fracture process and blade acceleration distance on cutting effect. Takeuchi [5] simulates the separation and fracture process of the spacecraft according to the shock response. Ren et al. [6] established a three-dimensional model of explosive cutting of CFRP composites and studied the influence of weakening groove on the cutting results of laminates. The results show that the existence of weakening groove can reduce the separation of laminates. Ochola et al. [7] studied the mechanical properties of the composite plates under compression load under different strain rates. The results showed that the failure modes of the composite plates in the lower layer of the low strain rate were mainly shear failure, while the failure modes of the composite plates at the high strain rate were mainly fiber and matrix debonding, base compression failure, and interlayer delamination.

In the past, the explosive separation of carbon fiber composite weave plate was usually carried out by the drilling charge method and the shaped charge cutting method. Considering the difficulty of drilling and the complex structure of shaped charge, a new separation method is needed. In this paper, based on the inertial confinement effect of metal shell on explosive energy in shell charge, copper and lead are, respectively, used as shell confinement explosive energy, and a charging structure of $\mathrm{C}$-shaped metal 
shell enclosed detonating cord is designed, as shown in Figure 1. The advantages of the metal shell are as follows. (1) Increase the detonation speed of explosives. The existence of shells can increase the detonation speed of explosives, and its effect is the same as increasing the diameter of the charge column. (2) It is conducive to stable detonation of explosives. Due to the existence of shells, radial diffusion in the detonation propagation process of explosives is limited to a certain extent, and radial sparse wave interference to the reaction zone is avoided. (3) Shell plays a limiting role on radial expansion of detonating gas, which extends the retention time of detonating gas in charge space, restricts radial expansion of detonating gas, and increases energy flow velocity.

Considering the characteristics of high specific strength, high specific stiffness, and good fatigue resistance of carbon fiber composite, the structure of charging at both sides of the plate is adopted to make the shock wave generated on both sides of the plate converge at the center of the plate after initiation, so as to improve the front pressure of the wave, improve the energy utilization rate, and facilitate the separation of the plate with explosive energy more effectively The advantages of the cutting device provide a reference scheme for explosive separation of small charge structure in narrow and complex environment.

\section{Numerical Simulation}

In order to fully understand the two types of metal shell in the process of explosive cutting of explosion energy of inertial confinement effect, now the of the finite element analysis program AUTODYN software analysis is used, considering the explosion in the process of explosives and plate can appear large deformation and breakage, so meshless SPH algorithm is used for the explosion of the carbon fiber woven composite plate separation for numerical simulation. In the SPH algorithm, the approximation of the function $f(x)$ and its derivative $\nabla \cdot f(x)$ is discretized as [8]

$$
\begin{gathered}
f\left(x_{i}\right) \approx \sum_{j} \frac{m_{j}}{\rho_{j}} f\left(x_{j}\right) W_{i j}, \\
\nabla \cdot f\left(x_{i}\right) \approx \sum_{j=1}^{N} \frac{m_{j}}{\rho_{j}} f\left(x_{j}\right) \cdot \nabla_{i} W_{i j} .
\end{gathered}
$$

Therefore, the conservation of mass, momentum, and energy in the SPH algorithm can be expressed as (without considering the body force)

$$
\left[\begin{array}{l}
\frac{d \rho_{i}}{d t} \\
\frac{d v_{i}^{a}}{d t} \\
\frac{d e_{i}}{d t}
\end{array}\right]=\left[\begin{array}{c}
m_{i} \cdot \sum_{j=1}^{N} v_{i j}^{b} \\
-\sum_{j=1}^{N} \frac{m_{j}}{\rho_{j}}\left(\frac{\sigma_{i}^{a b}+\sigma_{j}^{a b}}{\rho_{i}}+\prod_{i j}\right) \\
\frac{1}{2} \sum_{j=1}^{N} \frac{m_{j}}{\rho_{j}}\left(\frac{\sigma_{i}^{a b}+\sigma_{j}^{a b}}{\rho_{i}}+\prod_{i j}\right) v_{i j}^{b}
\end{array}\right] \cdot \frac{\partial W_{i j}}{\partial x_{i}^{b}}
$$

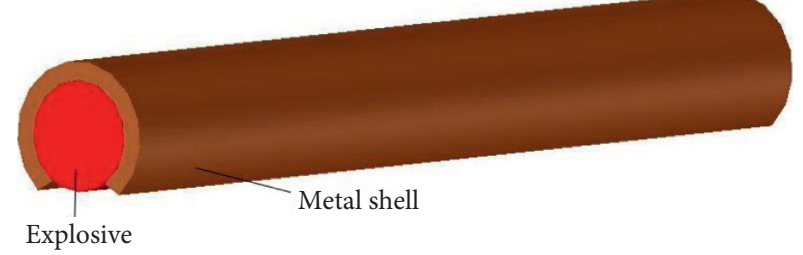

FIgURE 1: C-shaped shell charge structure.

where $\rho, m, v, e, t, x$, and $\sigma$ represent density, mass, velocity, energy, time, coordinate, and stress, respectively, $a$ and $b$ represent the axial direction, $W_{i j}$ is a smooth function of a pair of particles $a$ and $b$, and a cubic spline function is used in this article, and $\prod_{i j}$ indicates the artificial viscosity introduced in the calculation.

In view of the nonuniformity and anisotropy of the carbon fiber composite plate, its failure must consider the coupling effect of material compression and tension. Therefore, the stress value of the point under the explosion load is monitored through the analysis and calculation model during the simulation process, and combining with the Tsai- $\mathrm{Wu}$ tensor strength criterion [9], it is judged whether there is a fracture in the monitoring point area, which provides a reference for the subsequent explosion separation test.

2.1. Simulation Model. The size of the carbon fiber composite plate is $120 \mathrm{~mm} \times 120 \mathrm{~mm} \times 15 \mathrm{~mm}$, the radius of detonating cord is $3 \mathrm{~mm}$, and the thickness of lead and copper shell is $1 \mathrm{~mm}$. Three sets of simulation models were established, and the charge was placed symmetrically on both sides of the plate. Taking the side center of the plate as the coordinate origin, the horizontal direction is $X$ direction, the vertical direction is $Z$ direction, and the vertical $X O Z$ face inward is $Y$ direction. The first group uses exposed detonating cord for contact explosion, the second group wraps the detonating cord with $\mathrm{C}$-shaped copper shell, and the third group wraps the detonating cord with C-shaped lead shell. There are 4 monitoring points in each model group, and monitoring points $1 \sim 3$ are used to monitor stress values, which are located at the trisection of the central axis of the plate, at the coordinates $(0,0,0),(0,60,0)$, and $(0,120,0)$. Monitoring point 4 is used to calculate the specific internal energy value and velocity value of the measuring point area (the first group of models is not covered, so the monitoring points are set at the same coordinates), and the monitoring points are located at coordinates $(0,60,14)$. Use the function of automatic contact in AUTODYN to define automatic contact between materials. The bottom surface of the plate is fixed on the fixture, so displacement constraint is imposed on the whole bottom surface of the plate. Three simulation models are shown in Figure 2.

The dynamic response of materials when subjected to external loads is mainly manifested as deformation, flow, and fracture. The description of its dynamic response is a very complicated process, which is generally described by state equations and constitutive models [10]. The equation of 


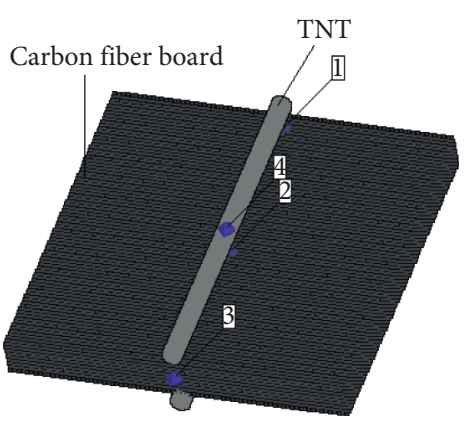

(a)

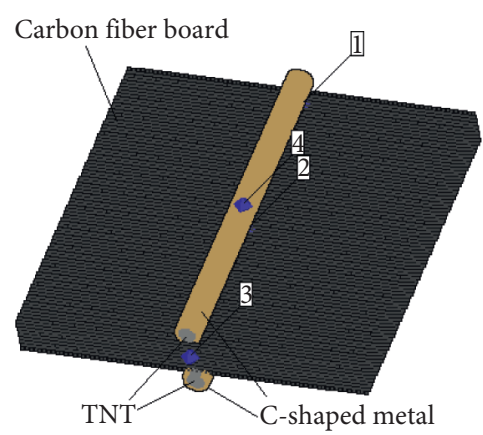

(b)

Figure 2: Numerical simulation model. (a) No metal shell constraint. (b) Metal shell constraint.

state of materials is a relationship related to pressure, density, and some thermodynamic parameters (internal energy and temperature), which reflects the volume characteristics of materials, while the constitutive model reflects the biased strain of materials, which is related to the strain, strain rate, internal energy, and grain size. The plate adopts orthotropic ortho constitutive model, orthotropic yield strength model, and orthotropic softening failure model, and its density is $1.85 \mathrm{~g} / \mathrm{cm}^{3}$, and the rest of the equation of state parameters are shown in Tables 1 and 2 .

The detonating cord is used as the main explosive medium to perform work. The pressure of the explosive detonation product is described by the JWL equation of state [11]. The JWL equation of state is widely used in the numerical simulation of detonation and explosion drive, and it is a form that does not contain chemical reaction to express the functional power of explosive detonation products [12], and its general expression is:

$$
P=A\left(1-\frac{\omega}{R_{1} V}\right) e^{-R_{2} V}+B\left(1-\left(\frac{\omega}{R_{2} V}\right)\right)+\left(\frac{\omega E}{V}\right),
$$

where $E$ is the initial specific internal energy, $V$ is the specific volume, $P$ is the pressure, $A, B, R_{1}, R_{2}$, and $\omega$ are the constants, and $R_{1}$ and $R_{2}$ are coefficients related to product expansion, generally set as $R_{1}=4.5$ and $R_{2}=1.5$. The rest of the equation of state parameters is shown in Table 3 . The first term on the right side of the equation represents the high pressure section, the second term represents the medium pressure section, and the third term represents the low pressure section. The effect of the first two terms of the equation in the late expansion of the detonation product can be ignored.

2.2. Model Validation. To ensure the effectiveness of the separation simulation method and the rationality of the separation model, the simple explosion separation test is used to verify the simulation model. In the verification test, a detonating cord is fixed on the surface of the plate, and the charge of the detonating cord is $12 \mathrm{~g} / \mathrm{m}$. In the test, the strain gauge is laid to monitor the strain value of the plate, and the strain monitoring points are set at the same position of the simulation model, and the results are compared. The strain values obtained from test and simulation are shown in Figure 3 . The trend of the two strain curves is basically the same, and the difference between the maximum strain values is only $6 \%$. This is because the fluctuation of shock wave will cause errors in numerical simulation, and the numerical model can reproduce the test results within a reasonable range, which proves the rationality of the simulation model.

2.3. Pressure Contour of Cross Section. Figure 4 shows the pressure contour of three groups of models at different times. It can be seen that the shock wave starts to enter the plate from both sides at $2 \mu s$, causing great compression effect on the plate. Moreover, two shock waves collide at the center of the plate at $4 \mu \mathrm{s}$. The frontal collision of two shock waves of the same intensity can be equivalent to the regular reflection of the shock wave on the solid wall, and the wave front at the center of the collision has the highest intensity; therefore, it is speculated that the initial maximum damage is located at the impact point. At $6 \mu s$, the first shock wave collision is completed, and the two reflected waves return to the original path. At $8 \mu s$, two reflected waves reach the boundary of the plate, respectively, and the sparse wave is reflected to the center of the plate at the boundary. In this process, the plate is subjected to the tensile action caused by the sparse wave. Comparing the pressure contour of the carbon fiber composite plate without shell constraint and with shell constraint, it can be seen that due to the existence of shell constraint, the shock wave front entering the plate is flatter, indicating that there is no obvious lateral sparse effect so that more explosion energy can be concentrated on the center of the plate.

2.4. Calculation Results and Analysis. The damage effect of three groups of models at different times is shown in Figure 5 . It can be roughly seen that the damage of model 1 is the smallest, that of model 2 is the second, and that of model 3 is the largest, and this is because the hard metal shell has a certain thickness and strength, when the explosive explodes; in addition to the transmission wave, there is also a compression wave reflected to the explosion center, which limits the movement of the explosive product in the nonopening direction of the shell and inevitably makes the energy 
TABLE 1: Structural parameters of the carbon fiber plate.

\begin{tabular}{lcccccccccc}
\hline$E_{1}(\mathrm{GPa})$ & $E_{2}(\mathrm{GPa})$ & $E_{3}(\mathrm{GPa})$ & $G_{1}(\mathrm{GPa})$ & $G_{2}(\mathrm{GPa})$ & $G_{3}(\mathrm{GPa})$ & $X_{t}(\mathrm{GPa})$ & $X_{c}(\mathrm{GPa})$ & $Y_{t}(\mathrm{MPa})$ & $Y_{c}(\mathrm{MPa})$ & $S(\mathrm{MPa})$ \\
\hline 52 & 46 & 8.9 & 6.0 & 4.8 & 6.2 & 1.86 & 1.15 & 216 & 408 & 102 \\
\hline
\end{tabular}

TABLE 2: Metal shell parameters.

\begin{tabular}{lcccc}
\hline Material & $\rho\left(\mathrm{g} \cdot \mathrm{cm}^{-3}\right)$ & $E(\mathrm{GPa})$ & $G(\mathrm{GPa})$ & $\mu$ \\
\hline Lead & 11.35 & 17 & 7 & 0.42 \\
Copper & 8.9 & 110 & 40 & 0.32 \\
\hline
\end{tabular}

TABLE 3: JWL state equation parameters of detonating cord.

\begin{tabular}{lcccccccc}
\hline$A(\mathrm{GPa})$ & $B(\mathrm{GPa})$ & $R_{1}$ & $R_{2}$ & $\omega$ & $\rho\left(\mathrm{kg} \cdot \mathrm{m}^{-3}\right)$ & $D\left(\mathrm{~m} \cdot \mathrm{s}^{-1}\right)$ & $E\left(\mathrm{~J} \cdot \mathrm{m}^{-3}\right)$ & $P_{C J}(\mathrm{GPa})$ \\
\hline 371 & 3.2 & 4.5 & 1.5 & 0.3 & 1000 & 6097 & $3.6 \times 10^{9}$ & 7.63 \\
\hline
\end{tabular}

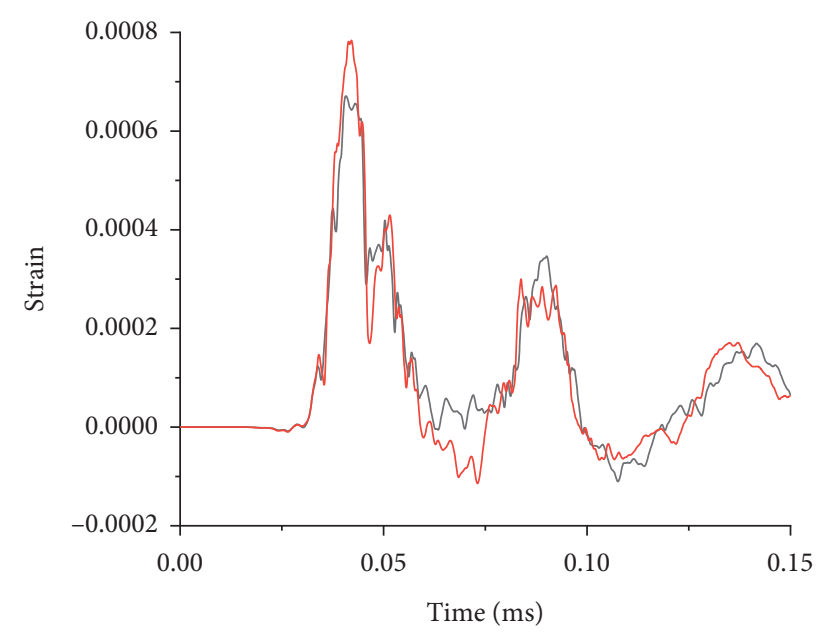

— Test

_ Simulation

Figure 3: Change of strain.
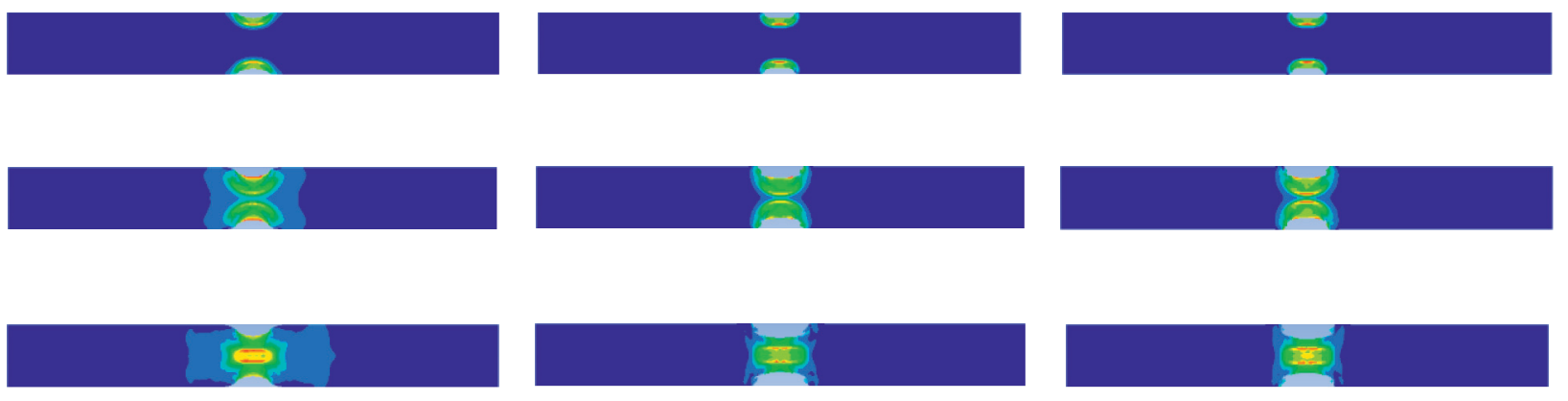

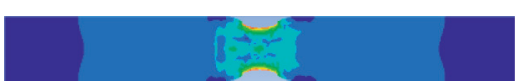

(a)

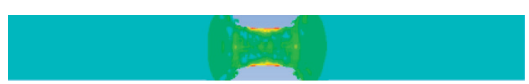

(b)

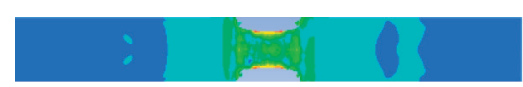

(c)

FIGURE 4: Pressure contour of each model cross section. (a) No metal shell constraint. (b) Copper shell constraint. (c) Lead shell constraint. 

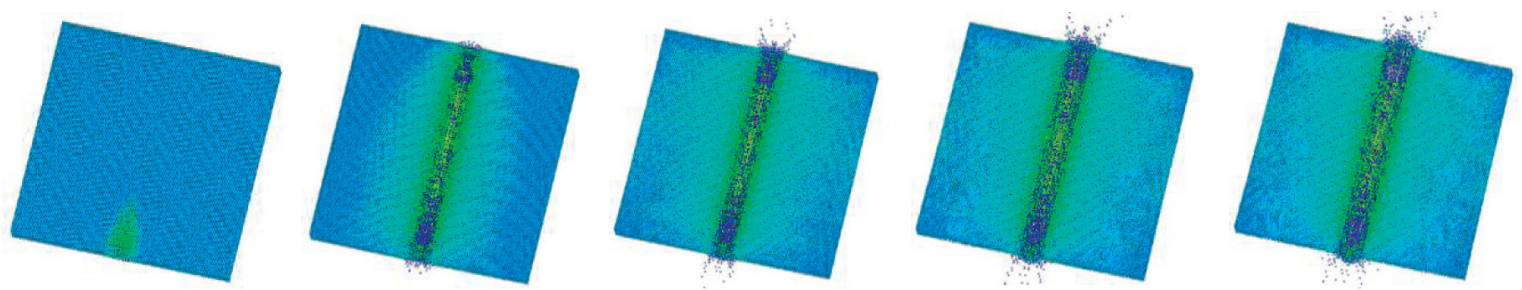

(a)
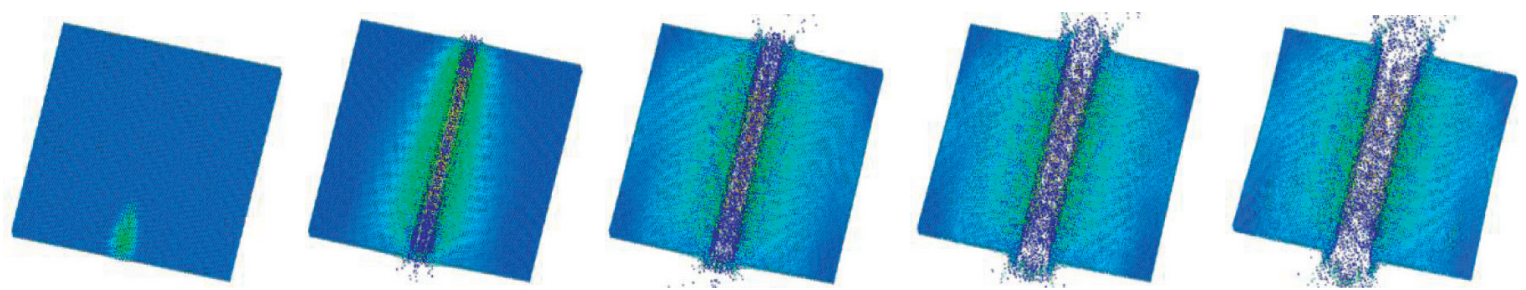

(b)
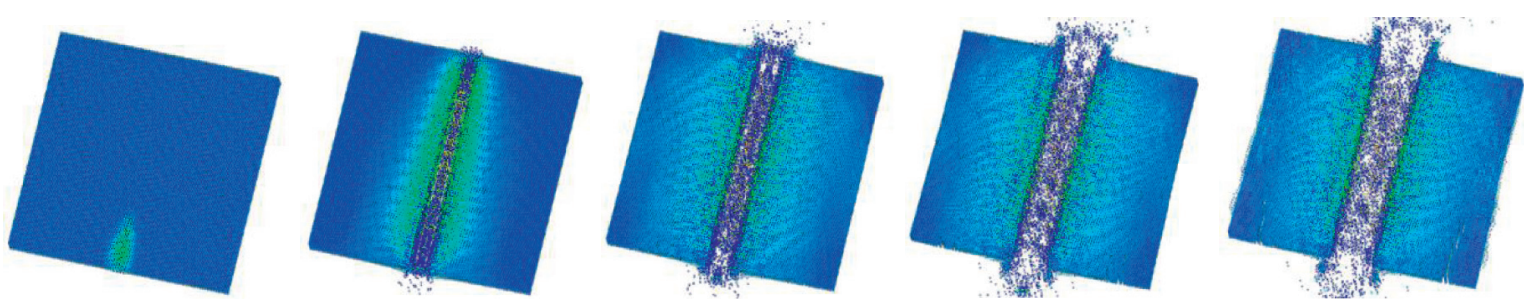

(c)

Figure 5: Damage state of each model. (a) No metal shell constraint. (b) Copper shell constraint. (c) Lead shell constraint.

converge to the opening of the C-shaped metal shell, forming the energy accumulation effect. Now, use the Tsai$\mathrm{Wu}$ tensor strength criterion to make a theoretical judgment on whether the single layer material is damaged.

Tsai-Wu tensor criterion polynomial:

$$
F=\left(F_{1} \sigma_{1}+F_{2} \sigma_{2}+F_{11} \sigma_{1}^{2}+F_{22} \sigma_{2}^{2}+F_{66} \tau_{12}^{2}\right)+2 F_{12} \sigma_{1} \sigma_{2}
$$

In formula (4),

$$
\begin{aligned}
& F_{1}=\frac{1}{X_{t}}-\frac{1}{X_{c}}=-3.32 \times 10-4 \mathrm{MPa}-1, \\
& F_{2}=\frac{1}{Y_{t}}-\frac{1}{Y_{c}}=-2.18 \times 10-3 \mathrm{MPa}-1, \\
& F_{11}=\frac{1}{X_{t} X_{c}}=4.68 \times 10-7 \mathrm{MPa}-2, \\
& F_{22}=\frac{1}{Y_{t} Y_{c}}=1.13 \times 10-5 \mathrm{MPa}-2, \\
& F_{66}=\frac{1}{s^{2}}=9.61 \times 10-5 \mathrm{MPa}-2, \\
& F_{12}=-\frac{1}{2} \sqrt{F_{11} F_{22}}=-1.15 \times 10-6 \mathrm{MPa}-2 .
\end{aligned}
$$

According to the Tsai-Wu tensor criterion, if the combination of the stress values of the monitoring points leads to tensor formula (4) $F \geq 1$, the plate in the monitoring point area will fracture. The larger the numerical value is, the greater the damage effect of explosion load is in the region of this point, where $F_{i j}$ is the strength parameter of the material. The stress values of three groups of simulated monitoring points are shown in Table 4.

By substituting the stress values of each group of monitoring points into formula (4), it is concluded that the $F$ values of the first group of monitoring points 1-3 are all less than 1 , and the model is not broken; the $F$ values of monitoring points 1-3 in the second and third groups are all greater than 1, so it can be judged that the model is completely broken. It can be preliminarily judged that the detonating cord without shell constraint cannot separate the carbon fiber composite plate, while the detonating cord under the metal shell constraint can separate the carbon fiber composite plate. It can be seen from Table 4 that the principal stress values of the third group of monitoring points are all greater than the corresponding principal stress values of the second group of monitoring points, which can indicate that the constraint effect of lead shell on explosive energy is better than that of copper shell.

The constraint effect of the two kinds of shells was compared by the incident wave energy of monitoring point 4. When the shock wave propagates from one medium to another, due to the different wave impedances of the two media, the shock wave will reflect and transmit at the interface of the two media. At the same time, the propagation process of the shock wave also needs energy maintenance. The energy reflected back to the original medium with the 
TABLE 4: Stress values of monitoring points.

\begin{tabular}{lccccccccc}
\hline & & & & & \multicolumn{3}{c}{$2^{\#}$ model } & \multicolumn{3}{c}{$3^{\#}$ model } \\
& 1point & 2point & 3point & 1point & 2point & 3point & 1point & 2point & 3point \\
\hline$\sigma_{1}(\mathrm{MPa})$ & 343 & 411 & 359 & 573 & 827 & 591 & 611 & 915 \\
$\sigma_{2}(\mathrm{MPa})$ & 257 & 326 & 278 & 410 & 650 & 453 & 425 & 798 \\
$\tau_{12}(\mathrm{MPa})$ & 67 & 84 & 74 & 98 & 135 & 106 & 117 & 152 & 121 \\
\hline
\end{tabular}

reflected wave is called the reflected wave energy, and the energy entering the other medium with the transmitted wave is called the transmitted wave energy. Monitoring point 4 observes the energy value of the monitoring point area; according to the conservation of energy, the energy of incident wave is equal to the sum of the energy of reflected wave and transmitted wave; the greater the energy of the monitoring point is, the greater the energy of corresponding transmitted wave will be, and the smaller the energy of reflected wave will be, the worse the shell constraint effect will be.

The energy distribution in the vicinity of the explosion can be expressed by the energy equation in the hydrodynamic equations. From the energy equation,

$$
\rho \frac{d}{d t}\left(U+\frac{V^{2}}{2}\right)=\rho F \cdot v+(\operatorname{div}(P v)+\operatorname{div}(k \operatorname{grad} T))+\rho q .
$$

It can be seen that the total energy of the explosion is composed of the sum of the kinetic energy and internal energy in the target medium and the explosive gas, which is the following formula:

$$
E=E e+E t=\left(E_{e k}+E_{e i}\right)+\left(E_{t k}+E_{t i}\right) .
$$

According to the energy distribution after shock wave, the total energy obtained after the medium is strongly impacted and is divided into specific internal energy and kinetic energy [13], so the sum of the specific internal energy and kinetic energy of the medium is the total energy of the transmitted wave. The curve of specific internal energy value at monitoring point 4 is shown in Figure 6, and the speed curve is shown in Figure 7.

It can be seen from Figure 6 and Figure 6 that the total energy of the transmitted wave changes with the change of the constraint conditions. It can be seen from Figure 6 that due to the existence of metal shell, the attenuation rate of curve (b) and (c) is much less than that of curve (a), and there is a second peak after the maximum peak value of curve (b) and (c). This is because at the nonopening of the $\mathrm{C}$-shaped shell, the detonation product directly impacts the inner wall of the shell, the density of the metal shell is greater than that of the detonation product on the detonation front, and the compressibility of the solid medium is generally less than that of the detonation product; the shock wave acting on the shell not only produces a small amount of transmitted wave but also reflects the compression wave to the explosion center. The reflected wave reflected from the shell for the first time propagates into the detonation gas, strengthening the internal pressure field, and there is a compression wave behind the sparse wave [14], which makes the energy in the shell increase again after reaching the shell. The same is true for the secondary velocity peak value of curve (b) and (c) in Figure 7. Extract the peak values of specific internal energy and speed at monitoring point 4 of each model and make Table 5.

From Table 5, we can get the energy of the transmitted wave: $E_{1}>E_{2}>E_{3}$, so the energy of the reflected wave: $E_{1}^{\prime}<E_{2}^{\prime}<E_{3}^{\prime}$, so the confinement ability of energy from air, copper to lead increases sequentially. The three sets of simulated explosive JWL equations of state are the same, so the initial strength of the shock wave is the same, and the deformation rate of the medium is negligible compared with the shock wave speed. Suppose the wave impedance of the grain is $\rho_{1} c_{1}$ and the wave impedance of the metal shell is $\rho_{2} c_{2}$; when the shock wave propagates from the grain to the shell, due to the different wave impedances of the media on both sides of the interface, the incident compression wave $\sigma_{I}$ will cause the reflected wave $\sigma_{R}$ and the transmitted wave $\sigma_{T}$ on the interface. $F$ represents the reflection coefficient and $T$ represents the transmission coefficient, which is obtained by the conservation of momentum and boundary conditions on the one-dimensional wave surface [15]:

$$
\begin{aligned}
& \left.\begin{array}{l}
\sigma_{R}=F \sigma_{T} \\
\nu_{R}=-F \nu_{T}
\end{array}\right\}, \\
& \sigma_{T}=T \sigma_{I} \\
& \left.v_{T}=\left(\frac{\rho_{1} c_{1}}{\rho_{2} c_{2}}\right) T v_{I}\right\} \text {, } \\
& F=\left(\frac{\rho_{2} c_{2}-\rho_{1} c_{1}}{\rho_{1} c_{1}+\rho_{2} c_{2}}\right) \\
& \left.T=\left(\frac{2 \rho_{2} c_{2}}{\rho_{1} c_{1}+\rho_{2} c_{2}}\right)\right\} \text {. }
\end{aligned}
$$

The wave impedance of copper is greater than that of lead, and the wave impedance of detonating cord is much smaller than that of lead and copper. Combined with formula (8), it can be seen that the transmission coefficient of lead shell is smaller than that of copper shell, so the transmission energy of lead shell is smaller than that of copper shell. This is consistent with the conclusion drawn from Figures 5 and 6 . Therefore, when the initial wave speed and intensity are the same, the reflected energy of the lead shell is the largest, followed by the copper shell, and the unconstrained reflected energy is the smallest.

It can be seen from formula (6) that the left side of the energy equation is formed by adding internal energy and 


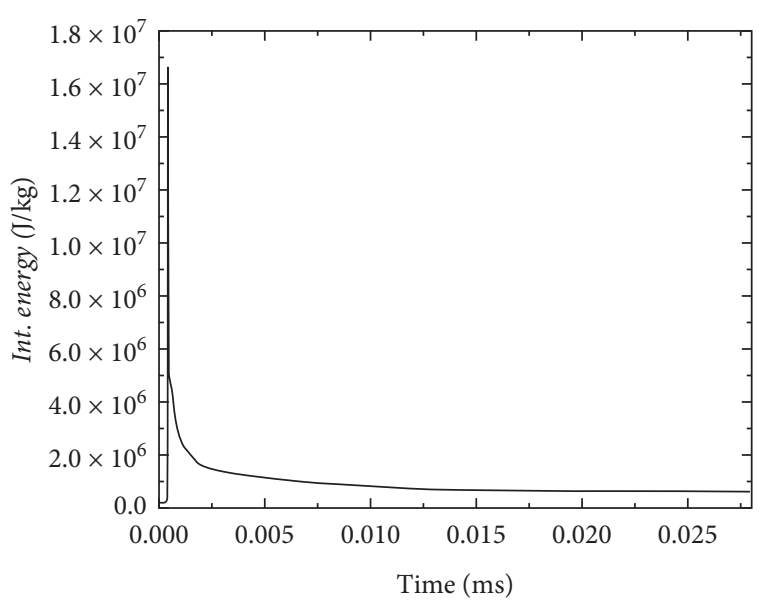

(a)

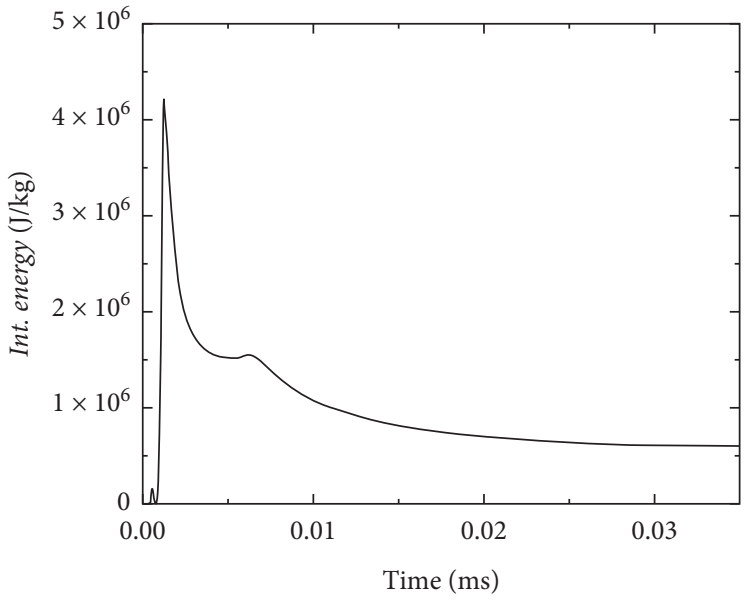

(b)

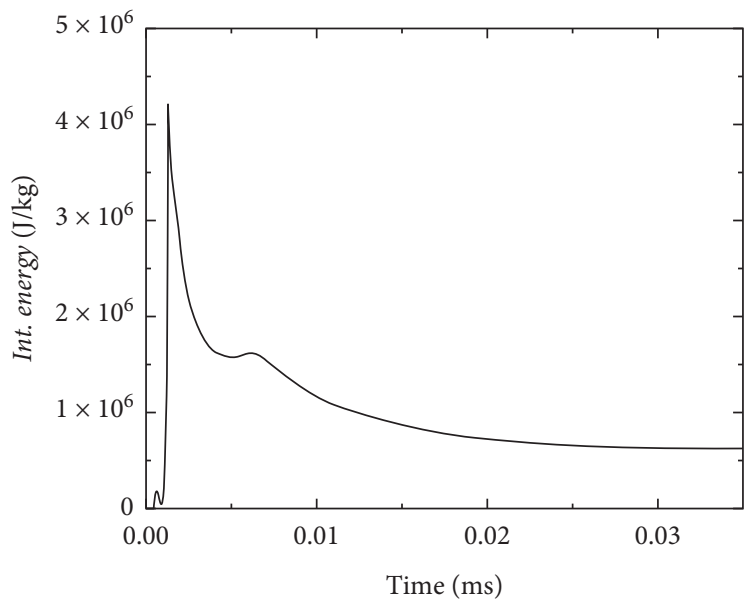

(c)

Figure 6: Monitoring point 4 specific internal energy curve. (a) No metal shell constraint. (b) Copper shell constraint. (c) Lead shell constraint.

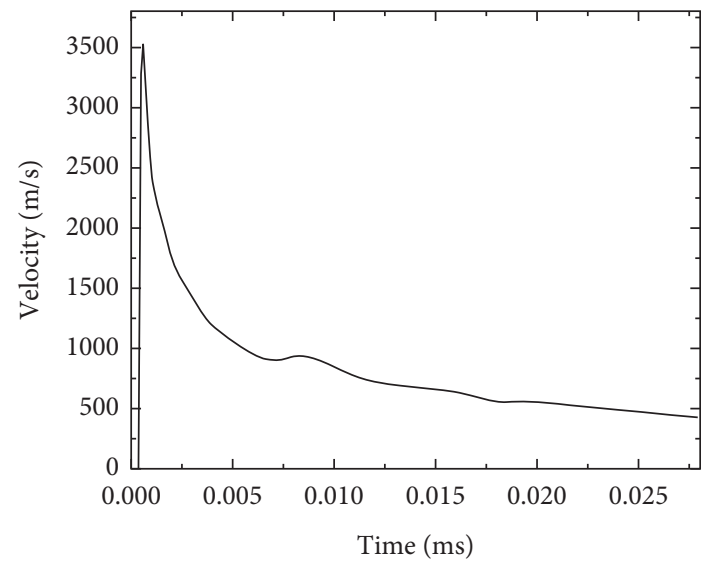

(a)

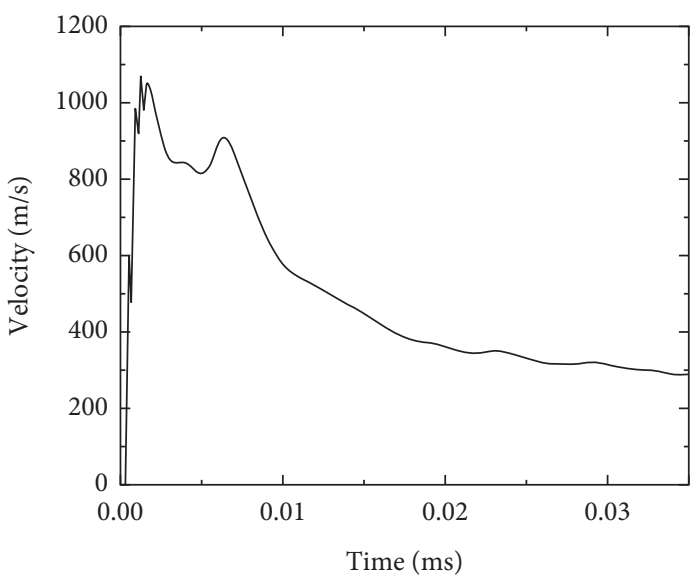

(b)

FIgURE 7: Continued. 


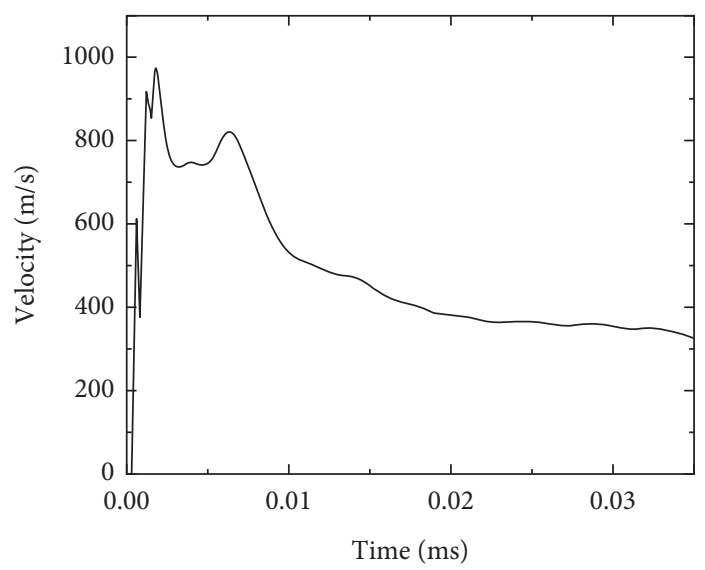

(c)

FIgURE 7: Monitoring point 4 speed curve. (a) No metal shell constraint. (b) Copper shell constraint. (c) Lead shell constraint.

TABle 5: Maximum specific internal energy and maximum velocity values at monitoring point 4 .

\begin{tabular}{lccc}
\hline & $1^{\#}$ model & $2^{\#}$ model & $3^{\#}$ model \\
\hline Maximum int. energy $\left(\mathrm{J} \cdot \mathrm{kg}^{-1}\right)$ & $1.60 \times 10^{7}$ & $4.21 \times 10^{6}$ & $4.19 \times 10^{6}$ \\
Maximum speed value $\left(\mathrm{m} \cdot \mathrm{s}^{-1}\right)$ & 3521 & 1070 & 976 \\
\hline
\end{tabular}

kinetic energy. Now, select the center section of the model and integrate the internal energy in the shell at each time point grid by grid to get the total amount of kinetic energy at each time point. Then, add the total amount of internal energy and kinetic energy at each time point to get the curve of total energy of the metal shell changing with time, as shown in Figure 8.

After the explosion shock wave reaches the metal shell for the first time, the energy in the shell rises rapidly and reaches its peak value under the action of the first transmission shock wave. At this time, the metal shell is in a high pressure state, and in the process of pressure propagation, the shock wave propagates outward when encountering the air interface and quickly takes away a large amount of energy. From the phenomenon, the energy decreases step by step after its peak value. In the initial stage, the metal shell is reflected back to the internal energy of the explosive gas, which strengthens the pressure of the explosive gas, and the secondary transmitted compression wave reaches the inner shell again, which greatly slows down the rate of the shell energy decline; therefore, a stepped platform appears in the curve. After the cumulative integral comparison of the two cases of copper shell and lead shell, it can be found that the energy in the copper shell is about 1.05 times higher than that in the lead shell; from this, it can be obtained that the energy absorption of the shell is copper $>$ lead, which further indicates that lead is a relatively good shell material for constraint energy.

\section{Carbon Fiber Composite Material Separation Test}

3.1. Experimental Design. The test plan was designed according to the simulation plan. The length, width, and

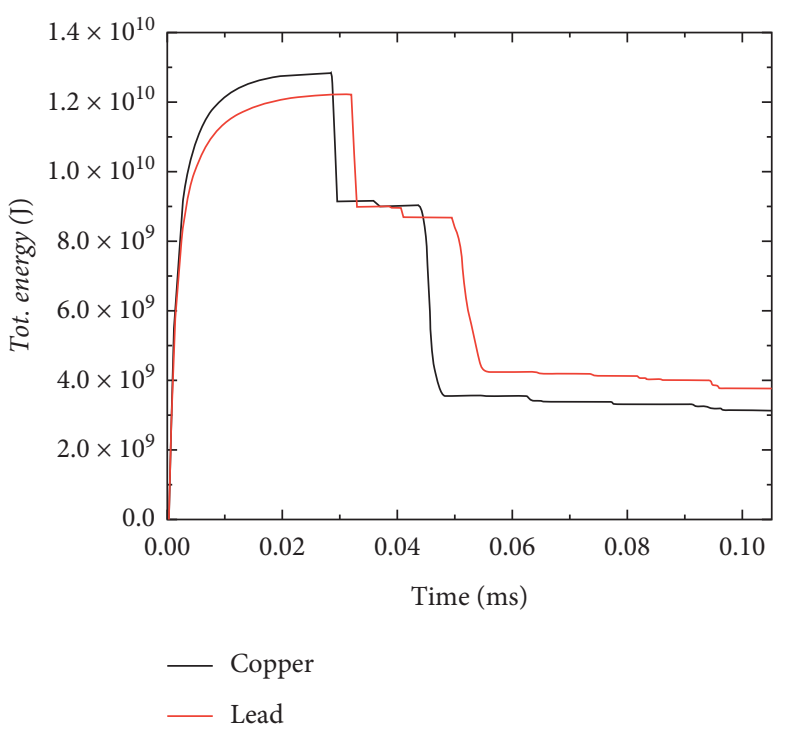

Figure 8: Total energy curve of metal shell.

height of the carbon fiber composite woven plate (corresponding to the $X, Y$, and $Z$ directions, respectively) are $120 \mathrm{~mm}, 120 \mathrm{~mm}$, and $15 \mathrm{~mm}$, as shown in Figure 9. The charge structure is to process the metal shell into a C-shape and insert the detonating cord inside.

There are three groups of tests, the first group is blank test, and the charging structure is shown in Figure 9(a), that is, two $200 \mathrm{~mm}$ detonating cords are symmetrically fixed on both sides of the plate along the $Y$ direction, and the detonating cords are located at the center line of the $X$ direction. The structure of the second group is shown in Figure 9(b), on the basis of the first group, the detonating cord is wrapped with a C-shaped lead shell with a thickness of $1 \mathrm{~mm}$, and the length of the lead is the same as the length of the plate $(120 \mathrm{~mm})$. The structure of the third group is shown in Figure 9(c), the lead shell of the second group is replaced with copper shell of the same size. The total mass of the two detonating cord used in each group of tests is $4.8 \mathrm{~g}$. In order to strictly control the simultaneous initiation of the 


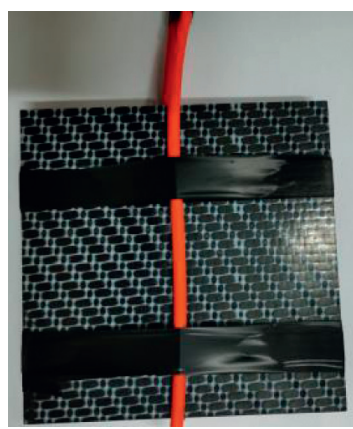

(a)

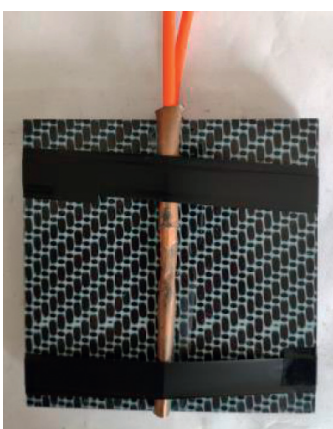

(b)

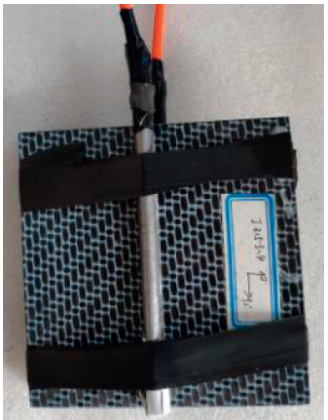

(c)

Figure 9: Charge structure. (a) $1^{\#}$ plate. (b) $2^{\#}$ plate. (c) $3^{\#}$ plate.

detonating cord on both sides of plates, the ends of the two detonating cord are bound and connected with a detonator. The device structure is shown in Figure 10.

3.2. Test Results. After the explosion reaction, collect the test plates and observe the results for comparison. The test results are shown in Figure 11.

It can be seen from the test phenomenon that the test effect of $1^{\#}$ plate without any inertial constraint is the worst, and the composite plate has the least damage and is not separated; $2^{\#}$ plate and $3^{\#}$ plate are completely separated. The tensile strength of the plate is less than the compressive strength; meanwhile, it can be seen from Figure 12(a) that the side damage effect of $1^{\#}$ plate is significantly layered in the direction of thickness, so the damage mode of $1^{\#}$ plate is mainly tensile failure caused by stress wave. It can be seen from Figures 12(b) and 12(c) that the fracture damage effect of $2^{\#}$ and $3^{\#}$ plates is different from the side damage of $1^{\#}$ plate, the fracture surface of $2^{\#}$ and $3^{\#}$ plates is relatively flat, and there is no stratification of matrix fibers in the direction of fracture thickness, and it can be judged that the $3^{\#}$ plate is directly broken under the one-time compression with strong explosion load, and the plate was already broken before the shock wave reached the free surface and underwent reflection stretching, so the damage mode of the $2^{\#}$ and $3^{\#}$ plates is mainly the compression damage of the shock wave. The failure mode is shown in Figure 13.

The surface of the $1^{\#}$ plate has a wider groove under the explosive load of the detonating cord. This is due to the existence of the C-shaped metal shell opening, which makes the shock wave and detonation products have a large energy flow density and concentrates in a small area, and its impulse density is greater than the critical impulse density of the target medium, and when it acts on the surface of the plate, it will cause local compression and matrix cracking. The shock wave propagates in the form of near spherical wave inside the composite plate; after the shock wave collides with the other side at the center, the wave front pressure increases, the fiber at the center is damaged first, and the shock wave continues to propagate to the other end of the plate to produce reflection stretching, and the tensile stress causes the fiber at the surface indentation crack to pull out, resulting in a wide groove on the surface of the

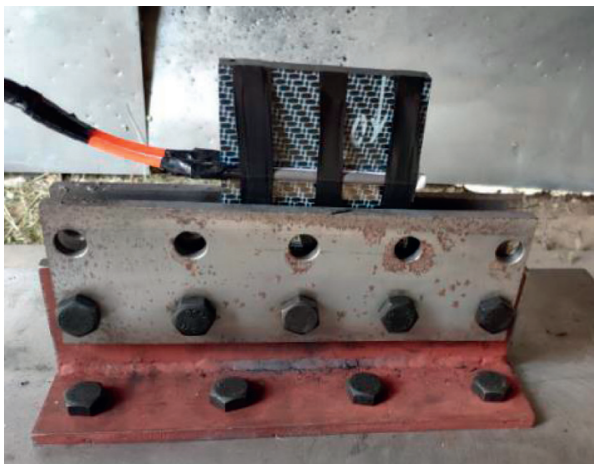

Figure 10: Device structure.

plate, as shown in Figure 11(a). At the same time, the residual stress wave pulse will reflect on the free surface formed by the central damage area; if the residual stress wave amplitude is high enough, tensile fracture will occur again near the damage surface [16], so as to form multiple spallation surfaces, as shown in Figure 12(a), and several obvious spallation surfaces appear along the direction from the center of the plate to both sides. This tensile stress strength cannot further damage the plate and cannot make it completely fracture, but can make the matrix fiber delamination.

$2^{\#}$ and $3^{\#}$ plates add shell constraint to detonating cord, when the explosion shock wave reaches the metal shell, it is reflected, and the reflected shock wave propagates into the explosion gas, the secondary compression wave formed by the sharp rise of the internal pressure of the explosion gas will catch up with the shock wave in the interface and composite plate, the peak pressure under the joint action of the two shock waves exceeds the compressive strength of the plates, and the plate is directly crushed under the strong compression action; it is too late to have reflection stretching phenomenon, so the fracture surface is relatively flat and there is no spalling phenomenon, as shown in Figures 12(b) and 12 (c). In addition, the shock wave speed is much larger than the shell deformation rate; after the secondary compression wave is reflected on the shell interface, it will also propagate into the explosive gas; however, the pressure formed inside the explosive gas is relatively small at this time, and its strength can be ignored. 


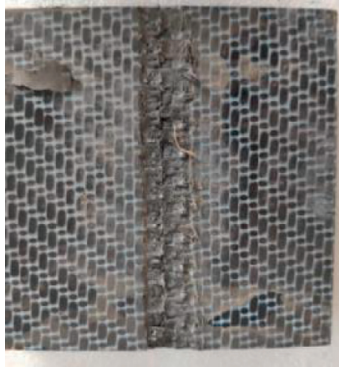

(a)

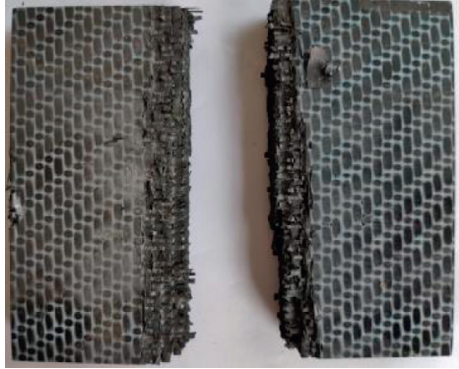

(b)

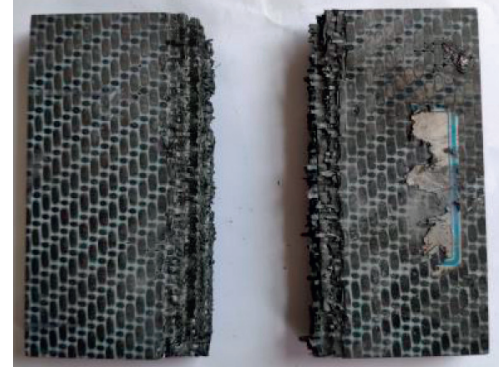

(c)

Figure 11: Test effect. (a) $1^{\#}$ plate. (b) $2^{\#}$ plate. (c) $3^{\#}$ plate.

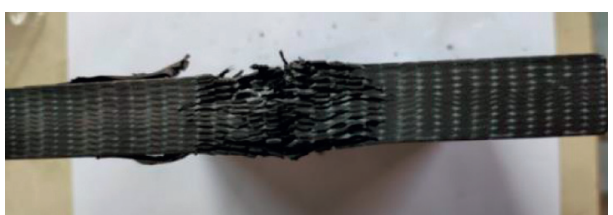

(a)

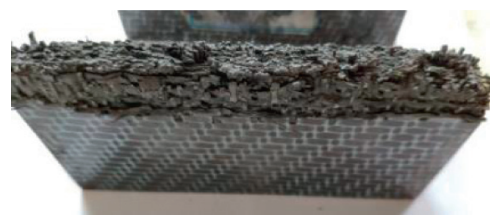

(b)

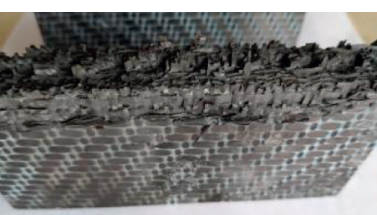

(c)

Figure 12: Plate damage. (a) $1^{\#}$ plate. (b) $2^{\#}$ plate. (c) $3^{\#}$ plate.
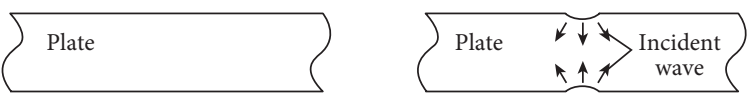

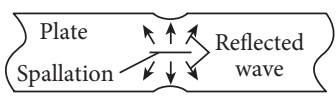

a)

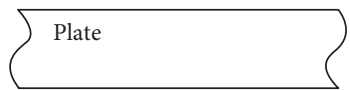

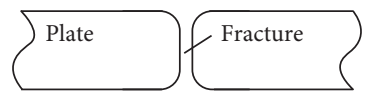

(b)

Figure 13: Plate failure mode. (a) No metal shell constraint. (b) Metal shell constraint.

The wave energy matching is actually the wave impedance matching of the media on both sides of the interface [17]. If the wave impedance ratio of the two media is larger, the reflected energy will be greater. The wave impedance ratio of detonating cord and lead is greater than that of detonating cord and copper, and the ductility of lead is better than that of copper; under the same impact load, the time for lead to maintain integrity is also stronger than that of copper, so it can be seen that lead has stronger inertial restraint ability on explosive energy and can better gather explosion energy. This is also consistent with the simulation results.

\section{Conclusion}

This article focuses on the explosive separation of carbon fiber composite woven plates under inertial constraint and combines numerical simulation with experiments. Explosive separation of the carbon fiber composite woven plate with a detonating cord wrapped in a metal shell successfully separated the composite plate that could not be separated without a shell, providing a reference plan for the explosive separation of the small charge structure in a narrow and complex environment. The following conclusions were obtained:
(1) The carbon fiber composite woven plate cannot be separated without the detonating cord wrapped by the metal shell, and the reason is that the shock waves on both sides collide at the center of the plate, although the wavefront pressure is increased and the inner center is damaged first, but the impact load strength cannot separate the plate, and the shock wave is reflected and stretched by the free surface, causing many layer cracks in the thickness direction of the plate center.

(2) The main reason for the fracture failure of the plate is the compression effect when the shock waves on both sides of the plate propagate to the center at the same time, and the layer cracks' failure is caused by the tensile action of stress wave in later period, under the premise that the plate has not been broken by impact. The charge structure of the detonating cord wrapped by the metal shell can effectively use its inertia to restrain the explosion energy and improve the separation ability during the explosion separation of the carbon fiber composite woven plate, and the effect of the lead shell to constraint the explosion energy is better than that of the copper shell. 


\section{Data Availability}

All data used to support the findings of this study are included within the article.

\section{Conflicts of Interest}

The authors declare that there are no conflicts of interest regarding the publication of this paper.

\section{Acknowledgments}

This work was sponsored by the National Natural Science Foundation of China (Grant no. 11002162).

\section{References}

[1] I. Whalley, "Development of the STARS II shroud separation system," in Proceedings of the Joint Propulsion Conference and Exhibit, San Jose, CA, USA, July 2013.

[2] T. Nicolas, "Tensile testing of materials at high rates of strain," Experimental Mechanics, vol. 21, no. 5, pp. 177-185, 1981.

[3] R. C. Hsiao, Analysis of Linear Shaped Charge Igniting Rapid Deflagration Cord Using Explicit Dynamics Method, American Institute of Aeronautics and Astronautics, Reston, VA, USA, 2015.

[4] S. Xiong, Y. Ye, Y. Li, Y. Wen, and H. Hassan, "Numerical study on the explosive separation of pyrotechnic cutter," Shock and Vibration, vol. 2019, Article ID 2457854, 13 pages, 2019.

[5] S. Takeuchi and J. Onoda, "Estimation of separation shock of the marman clamp system by using a simple band-mass model," Transactions of the Japan Society For Aeronautical And Space Sciences, vol. 45, no. 147, pp. 53-60, 2002.

[6] M. Ren, F. Weng, J. Sun, K. Tang, L. Feng, and R. Chen, "Influence of weakening groove on cutting results of composites subjected to shaped charge jet," Shock and Vibration, vol. 2021, Article ID 5528574, 12 pages, 2021.

[7] R. O. Ochola, K. Marcus, G. N. Nurick, and T. Franz, "Mechanical behaviour of glass and carbon fibre reinforced composites at varying strain rates," Composite Structures, vol. 63, no. 3-4, pp. 455-467, 2004.

[8] G. R. Liu and M. B. Liu, "Smoothed particle hydrodynamics-a meshfree particle method," Computational Mechanics, vol. 33, no. 6 , p. $491,2003$.

[9] W. T. Stephen and M. W. Edwrd, "A general theory of strength for anisotropic materials," Journal of Composite Materials, vol. 5, no. 1, pp. 58-80, 1971.

[10] Livermore Software Technology Corporation (Lstc), LSDYNA: Keyword User's Manual-Volume I: Material Models, CAL, Chennai, India, 2012.

[11] G. Baudin and R. Serradeill, "Review of jones-wilkins-lee equation of state," EPJ Web of Conferences, vol. 10, 2010.

[12] J. B. Men, J. W. Jiang, and S. Y. Wang, Technical Basis for Numerical Simulation of Explosion Shock, Beijing Institute of Technology Press, Beijing, China, 2015.

[13] weixin Li, One Dimensional Unsteady Flow and Shock Wave, National Defense Industry Press, Beijing, China, 2003.

[14] M. Wang, T. Ma, and J. Ning, "Study on the release law of explosive energy in explosive concrete," Chinese Journal of High Pressure Physics, vol. 26, no. 5, pp. 517-522, 2012.

[15] Z. Wang, Y. Liu, J. Zhang et al., "Experimental study on the influence of constraint conditions and charge diameter on explosive pressure of HMX/F2641 booster," Explosion and Shock, vol. 23, no. 3, pp. 57-61, 2003.

[16] Орленко, Explosion Physics (Volume 2), Science Press, Beijing, China, Translated by Sun Chengwei, 2011.

[17] A. K. Umesh, "Wave energy conversion under constrained wave-by-wave impedance matching with amplitude and phase-match limits," Applied Ocean Research, vol. 90, 2019. 